\title{
Favoreciendo el cambio emocional positivo hacia las ciencias en la formación inicial del profesorado
}

\author{
Carolina Pipitone Vela; Fina Guitart Mas; Carlos Agudelo Carvajal; \\ Àngela García Lladó
}

Departament d'educació lingüística i literària i de didàctica de les ciències experimentals i de la matemática. Universitat de Barcelona. Barcelona, España.

[Recibido el 23 de octubre de 2018 aceptado el 5 de febrero de 2019]

En este trabajo identificamos las emociones de los futuros maestros (FM) en relación con la física y la química, además de buscar evidencias de que estas sean modificadas a lo largo del contexto de la asignatura Didáctica de la Materia, la Energía y la Interacción (DMEI). Después de pasar un cuestionario a los FM sobre sus emociones antes y después de la asignatura, los resultados muestran una modificación notable de emociones negativas hacia emociones positivas. Dichos cambios se relacionan con la metodología de trabajo implementada en la asignatura, concretamente con el carácter experimental que tiene. Los resultados también sugieren el reto de favorecer que los FM valoren la importancia que tienen los aspectos emocionales del aprendizaje, así como el hecho de dar sentido a los conocimientos.

Palabras Clave: Emociones, Formación docente, Física, Química, Educación primaria.

\section{Promoting the positive emotional change towards sciences, in the initial teacher training}

The aim of this work is to identify the emotions of the future teachers (FM) towards physics and chemistry, as well as look for evidences of their modification throughout the context of the subject 'Didactics of Matter, Energy and Interaction (DMEI). The responses of a questionnaire passed to the FM about their emotions before and after coursing the subject show a remarkable modification of negative emotions towards positive emotions. These changes are related to the methodology implemented in the subject, specifically with the experimental character that it has. The results also suggest the challenge that the FM value the importance of the emotional aspects of the learning, as well as to give sense to the knowledge they learn.

Keywords: Emotions, Teacher training, Physics, Chemistry, Primary education

Para citar el artículo. Pipitone, C. Guitart, F., Agudelo, C. y García Lladó, A. (2019). Favoreciendo el cambio emocional positivo hacia las ciencias en la formación inicial del profesorado. Ápice. Revista de Educación Científica, 3(1), 41-54. DOI: https://doi.org/10.17979/arec.2019.3.1.4608

Contacto.cpipitone@ub.edu, Fina.guitart@ub.edu, agudelocar@ub.edu,Angela.garcia@ub.edu 


\section{Introducción}

Año tras año, a la asignatura "Didáctica de la Materia, la Energía y la Interacción" (DMEI) de segundo año del grado de Maestro de Educación Primaria de la Universitat de Barcelona (y del doble itinerario de Infantil y Primaria) llegan estudiantes con actitudes negativas, poca motivación y gran inseguridad hacia la física y la química, así como hacia la idea de verse enseñándolas a sus futuros alumnos. Además, la mayoría de las veces presentan un dominio del contenido científico deficiente en relación con los cursos que han recibido durante su escolaridad.

El grupo de profesores de la asignatura DMEI, siendo conscientes de la necesidad y oportunidad que tenemos de revertir o al menos atenuar esta preocupante situación, hemos concentrado nuestros esfuerzos en dos frentes que podrían influir en las emociones de los futuros maestros (FM): diseñar las clases para que los alumnos se acerquen a una actividad científica escolar genuina y tratar aspectos emocionales de manera explícita en algunas clases.

La asignatura de DMEI se imparte en los laboratorios de física y química de la Facultat d'Educació y los bloques temáticos están estructurados alrededor de prácticas que realizamos de manera dialógica (Pipitone et al., 2016) y con distintos enfoques didácticos (Predicción, observación, Explicación -POE- Investigación dirigida, etc.), favoreciendo siempre un clima de confianza y respeto en el que los estudiantes puedan debatir sus ideas y sean protagonistas de las actividades. Mediante estas actividades dialógicas (AD) les ofrecemos ejemplos de cómo formular preguntas investigables y guiar un diálogo para contrastar ideas entre pares. Paralelamente, como explicaremos en la metodología, generamos discusiones y reflexiones sobre la importancia de los aspectos emocionales en la ciencia, específicamente en el contexto de la educación y de sus propias sensaciones con respecto a la asignatura.

Teniendo en cuenta nuestra posible incidencia en la disposición emocional de los FM frente a las ciencias, hemos diseñado un instrumento para que ellos puedan expresar sus emociones antes y después del curso, así como las causas que creen que han influido en la modificación de dichas emociones, si es que estas han cambiado, durante el transcurso de la asignatura.

En este contexto realizamos el estudio que presentamos aquí, cuya finalidad es identificar las emociones, relacionadas con la física y la química, que explicitan los estudiantes de segundo curso de grado de Maestro en Educación Primaria de la Universitat de Barcelona en el contexto de la asignatura DMEl; así como determinar si estas emociones son modificadas e identificar cuáles son las causas del cambio en caso de haberlo.

\section{Marco teórico}

Según Hargreaves (1998), las emociones forman parte del corazón de la enseñanza; incluyen sus cualidades más dinámicas, ya que son fundamentalmente el motor del aprendizaje. Esto puede explicar el rol destacado que tienen las emociones en el contexto escolar, con un impacto especial en la construcción de la "identidad profesional" de los futuros maestros (Shapiro, 2010; Zembylas, 2003).

En la literatura del área, en la última década (Dávila Acedo; Cañada; Sánchez Martín, y Mellado Jiménez, 2016; Mellado et al., 2014; Vázquez Alonso y Manassero-Mas, 2008), se hace referencia a las actitudes negativas que tienen los estudiantes hacia las asignaturas de ciencia. Tal y como mencionan Murphy y Beggs (2003), se evidencia un punto de inflexión en sus actitudes hacia la ciencia al concluir la educación primaria, aumentando el desinte- 
rés, el aburrimiento y las experiencias asociadas al fracaso. Esta misma perspectiva es compartida por diversas investigaciones (Osborne, Driver, y Simon, 1998; Parkinson, Hendley, Tanner, y Stables, 1998; Ramsden, 1998; Vázquez Alonso y Manassero-Mas, 2008).

Si bien nuestro objeto de estudio no son las niñas y niños sino sus FM, se da el caso de que aproximadamente el $70 \%$ de estos $\mathrm{FM}$ han hecho ciencias por última vez en la Educación Secundaria Obligatoria (ESO) y por lo tanto es razonable suponer que su actitud y emociones hacia las asignaturas de física y química han sido formadas en gran medida en esa etapa educativa. Además, los FM, quieran o no, tendrán una influencia de primera mano en la valoración de las ciencias que tengan sus alumnos, y por esto es muy importante que sean conscientes de la oportunidad que tienen para promover una valoración positiva de éstos hacia la ciencia.

\section{Las emociones y el dominio del contenido}

Tal como afirmamos en la introducción, durante la formación de los FM nos encontramos que los alumnos acceden a la asignatura con un dominio insuficiente del contenido científico y eso nos lleva a preguntarnos ¿Qué sucede con las emociones cuando no se dominan ciertos contenidos de la asignatura? Diversos estudios hacen referencia a la importancia que tiene el dominio del conocimiento científico en el desempeño del profesorado. Por ejemplo, Cañal (2000) señala que la escasa formación en contenidos científicos puede generar "analfabetismo funcional" respecto a la cultura científica, lo que supone una limitación para la enseñanza y una barrera para el cambio didáctico, ya que el profesorado se muestra más inseguro y con menos confianza en la enseñanza de las ciencias (Mellado, V., 2003).

Como consecuencia de esta inseguridad el profesorado se vuelve más dependiente del libro de texto, dedica menos tiempo de clase a aquellos contenidos que no domina y le resulta más difícil detectar las ideas alternativas y/o las dificultades de aprendizaje (Appleton, 1995; Harlen y Holroyd, 1997). En términos de Gess-Newsome (2002) reemergen los comportamientos de un profesorado novato; es decir, le dedica más esfuerzo a la gestión de la clase que al acompañamiento de los estudiantes en el proceso de aprendizaje (Hollon, Roth, y Anderson, 1991). Como sostiene Talanquer (2004), que un docente pueda crear las condiciones que favorezcan el aprendizaje no sólo depende del conocimiento del tema que enseña o de los métodos con que lo hace, sino que su éxito también parece depender de su capacidad para transformar el conocimiento disciplinario que posee en formas que resulten significativas para sus estudiantes. Esta transformación pedagógica del conocimiento científico requiere que el docente domine la materia y los procesos de construcción de dicho conocimiento.

Estos aspectos no son triviales ya que favorecen el desarrollo de un círculo vicioso que, por un lado, reproduce clases tradicionales, transmisoras y poco conectadas con la realidad debido a la falta de dominio del contenido por parte de los docentes y, por otro lado, clases poco motivadoras, generalmente basadas solamente en el libro de texto y cargadas de inseguridad. Estos dos aspectos condicionan a los FM y a sus alumnos a perpetuar el desagrado hacia las asignaturas de ciencias y a mantener el ciclo que bloquea su aprendizaje. Es interesante señalar al respecto que, en estudios recientes con futuros profesores, se ha encontrado que el recuerdo de las emociones vividas en secundaria se transfiere a su práctica docente (Borrachero Cortés, 2015).

\section{¿Por qué nos interesan las emociones de los FM?}

Nos proponemos abordar las emociones desde una "perspectiva socio-constructivista" concebidas como resultados de relaciones culturales, sociales e ideológicas (Hugo, Sanmartí, 
y Adúriz-Bravo, 2013; Zembylas, 2003). Desde este marco de referencia, Zembylas (2001) sostiene que las emociones, que los maestros experimentan y expresan, se construyen en sus relaciones sociales y en los sistemas de valores en sus familias, culturas y contextos escolares. Así pues, estas relaciones sociales y valores influyen profundamente en cómo y cuándo se construyen, expresan y comunican las emociones individuales. Por este motivo consideramos relevante incidir también en su construcción desde la formación de FM. Para ello debemos hacer explicitas las emociones de los FM durante la asignatura con la finalidad de que sean conscientes de ellas, reflexionar, y a partir de esta reflexión abordar la necesidad de cambio.

Además, coincidimos con la propuesta de Mega, Ronconi, y De Beni (2014) de que las emociones positivas en los estudiantes favorecen la autorregulación del aprendizaje, así como también influyen en la motivación y el rendimiento académico. Motivo que nos reafirma en la necesidad de incorporar las emociones en el planteamiento de nuestras propuestas didácticas.

\section{Objetivos}

Los objetivos planteados para este trabajo son:

- Identificar y explicitar las emociones de los FM en relación con la física y la química antes de comenzar la asignatura de DMEI.

- Identificar y explicitar las emociones de los FM en relación con la física y la química después de cursar la asignatura de DMEI.

- En caso de que haya modificación de las emociones, identificar y explicitar las causas que argumentan el cambio.

- Reflexionar sobre el papel de explicitar las emociones respecto a la física y la química y la planificación de momentos para hacerlo en clase.

\section{Metodología}

\section{Metodología de la investigación}

Este trabajo se comprende y enmarca dentro del paradigma cualitativo, ya que ha sido planteado con la finalidad de comprender la realidad dentro de un contexto determinado. La metodología cualitativa nos permite comprender la realidad del aula frente a una determinada situación, en este caso los aspectos emocionales que intervienen en las asignaturas de ciencias en la formación inicial de profesorado. Pueden establecerse inferencias y, a partir de estas, plantearse categorías de análisis que permiten entender (o interpretar) la realidad, con el fin de conseguir una coherencia lógica en el sucederse de los hechos o de los comportamientos que están necesariamente contextualizados, y es en el contexto donde adquieren su significado (Pérez Serrano, 1994)

Los datos analizados corresponden a las respuestas voluntarias de los cuestionarios sobre emociones y ciencia realizado en 6 grupos de clase de la asignatura DMEI del curso 20162017 impartida por 2 profesores. De estos grupos se obtienen 102 respuestas en la etapa inicial de la investigación y 104 respuestas al cuestionario que se contestan de manera voluntaria al finalizar la asignatura. Una vez identificadas las correspondencias entre los formularios PRE/POST, obtenemos un total de $56 \mathrm{FM}$ que han respondido a ambos.

El método de recogida de datos se lleva a cabo en dos etapas, una inicial en la que se pretende recuperar información sobre las emociones que explicitan los FM relacionadas a la física y la química antes de comenzar la asignatura, y una etapa final en la que se recoge la misma información después de haberla cursado. 
La recolección de datos correspondiente a la primera etapa se realiza durante la semana previa al inicio de clases, mediante un formulario digital a través del Campus Virtual. La segunda etapa de recolección de datos se lleva a cabo una semana antes de finalizar la asignatura, para ello se pasa un segundo formulario con las mismas preguntas generales del formulario inicial y se añaden algunas que hacen referencia a la argumentación del cambio (o no) de emociones que han experimentado.

El instrumento de recogida de datos está constituido por una seria de preguntas generales como, por ejemplo: género, modalidad del bachillerato/ciclo formativo cursado, edad, etc. Además, contiene preguntas específicas y relevantes para el estudio de las emociones y la experiencia vivida alrededor de las ciencias. La pregunta de la que sacamos la información sobre las emociones que siente el alumnado es cerrada: "Cuál de estas emociones vienen a tu cabeza cuando piensas en física y química. Elige tres". Y se les adjunta un listado de 24 emociones distintas para que seleccionen su respuesta. A parte, hay preguntas abiertas como por ejemplo “¿Qué expectativas tienes de la asignatura?"

En el proceso de análisis de los datos se utilizan, para una parte de ellos, las categorías propuestas por Mellado et al. (2014). A saber, un grupo de emociones positivas: orgullo, tranquilidad, placer, simpatía, entusiasmo, confianza, satisfacción, capacidad, diversión, motivación, interés y curiosidad; y un grupo de emociones negativas: ira; miedo; tensión; preocupación; odio; ansiedad; desesperación; nerviosismo; impotencia; frustración; aburrimiento y agobio. Nosotros hemos agregado sorpresa a las positivas e inseguridad a las negativas.

Posteriormente, en un segundo nivel de análisis, se identifican los argumentos que han utilizado los FM para justificar su cambio (o no) de emociones y con ellos se construyeron categorías de análisis.

Esta etapa de la investigación se ha llevado a cabo a partir de la comparación constante de los datos (Tesch, 1990). El método de comparación y contrastación es utilizado en los procesos de análisis como, por ejemplo, la formación de categorías, el establecimiento de los límites de estas, lo que resume el contenido de cada categoría, etc., con el objetivo es discernir similitudes conceptuales, refinar la capacidad discriminativa de las categorías, y así establecer patrones. Teniendo en cuenta estas características que definen el método de comparación hemos realizado el análisis de los datos, la elaboración y justificación de categorías.

Las categorías que surgen de los datos son cuatro, dos de ellas hacen referencia explícita a las metodologías de aula utilizadas y otras dos a aspectos más relacionados con dinámicas afectivas entre el docente y el grupo de clase. Dichas categorías las definimos de la siguiente manera:

- La metodología de aula como principal agente motivador del cambio: hace referencia a las dinámicas de trabajo en el aula que se fundamentan en las AD.

- El trabajo experimental y manipulativo como agente facilitador del cambio emocional: Hace referencia a la relevancia que tiene la parte experimental y manipulativa a la hora de trabajar una ciencia cercana y accesible para el alumnado.

- Los vínculos docente-alumno como promotor del cambio emocional: Hace referencia a la relación de confianza y seguridad que se establece en el aula.

- La despenalización del error como herramienta facilitadora del cambio emocional: Hace referencia al clima de respecto, confianza y seguridad que se crea en el espacio del laboratorio para poder hacer explicitas aquellas ideas erróneas que tienen los FM y que es necesario explicitar para poder avanzar en la construcción del conocimiento 
Estas categorías nos permiten analizar cuáles son los agentes motivadores del cambio emocional hacia las ciencias en los FM.

\section{Metodología de trabajo en el aula}

Consideramos relevante hacer explicita la metodología de trabajo en el aula, en relación con las emociones, ya que esto nos permite hacer una reflexión coherente entre lo que expresa el alumnado, en cuanto a las razones por las cuales cree que ha cambiado (o no) su disposición frente a la física y la química, como también al proceso que vive durante el curso de la asignatura.

A partir de las respuestas obtenidas del primer formulario se elabora una nube de palabras, para trabajar el primer día de clase, en la que destacan aquellas emociones que tuvieron más presencia en sus respuestas. A partir de la imagen se reflexiona con los FM sobre las emociones y sobre la implicación que pueden tener en el proceso de enseñanza-aprendizaje de ellos mismos como de sus futuros alumnos. De esta manera se hace explicita la inseguridad y la percepción que tienen los FM sobre su experiencia en clases de ciencia y sobre cómo estas emociones pueden condicionar su desarrollo profesional en el aula.

Como se sabe, en los procesos de enseñanza-aprendizaje tiene especial relevancia la comunicación, por ello las clases de DMEl se caracterizan por ser fundamentalmente experimentales a partir de las cuales se fomentan las AD entre los docentes y los FM. Por medio de las $A D$, trabajamos para favorecer esta construcción del conocimiento de los FM, a partir de las discusiones y reflexiones que se llevan a cabo en el aula de ciencias (Pipitone et al., 2016a).

Entendemos que trabajar con AD, favorece la evolución del conocimiento científico a partir de: diálogo, las preguntas que llevan a confrontar las ideas previas de los estudiantes, las miradas desde diferentes perspectivas de un mismo fenómeno. Todo ello nos permite conseguir esa evolución del conocimiento, teniendo como herramienta principal el diálogo reflexivo (Pipitone et al., 2016b).

Las AD no son las únicas metodologías de aula utilizadas en la asignatura, pero sí tienen un peso considerable. Las clases como hemos mencionado anteriormente son experimentales, y en estas prácticas utilizamos metodologías como las conocidas POE mediante las cuales también se ponen en práctica las AD. Otras de las metodologías utilizadas son las investigaciones dirigidas, prácticas que también favorecen el dialogo entre los diferentes participantes del aula.

Este tipo de metodologías dialógicas permiten que los FM vayan sintiéndose cómodos y cada vez con más confianza para expresar sus ideas con relación a los conceptos que se están trabajando en cada momento.

En el transcurso de la asignatura, y mediante las AD que se llevan a término en ellas, se van aprovechando las diferentes sesiones de aula para hacer explícitas las emociones que les pueden generar si les ha resultado difícil el aprendizaje, o si se han sorprendido al poder dar una explicación científica a una situación de la vida cotidiana.

La semana previa a la finalización del curso se les pide que respondan al segundo formulario de manera voluntaria y a partir de estos datos se elabora una nueva nube de apalabras en la que se recogen las emociones más mencionadas. De esta manera, en la última sesión del curso se repite la actividad realizada al comienzo, en primer lugar, recordando a los FM cuál era su relación emocional con la ciencia, especialmente con la física y la química; $y$, posteriormente, se les muestra la nueva nube de palabras con sus emociones actuales. 
A partir de estas imágenes se reflexiona sobre los cambios que han tenido con relación a su manera de ver y entender la ciencia, así como también sobre cuáles han sido los agentes que han favorecido ese cambio de actitud.

\section{Resultados}

A continuación, se presentan los resultados más destacados de la investigación en tres apartados: las emociones antes de comenzar DMEI, las emociones al acabar DMEI y la justificación de los cambios.

\section{Etapa inicial: antes de comenzar DMEI}

Los resultados más relevantes que hemos obtenido (Fig. 1) muestran un considerable predominio de las emociones negativas (representadas de color rojo) frente a las positivas (representadas de color azul), según las categorías propuestas por Mellado et al. (2014). Dentro de las emociones más mencionadas en los cuestionarios analizados, la primera es la inseguridad (en un $60 \%$ de las respuestas), seguida por la curiosidad (36\%). En tercer lugar, aparecen la motivación y el miedo $(23,6 \%)$, seguidas por la ansiedad y la frustración (20\%) y la confianza $(18,2 \%)$.

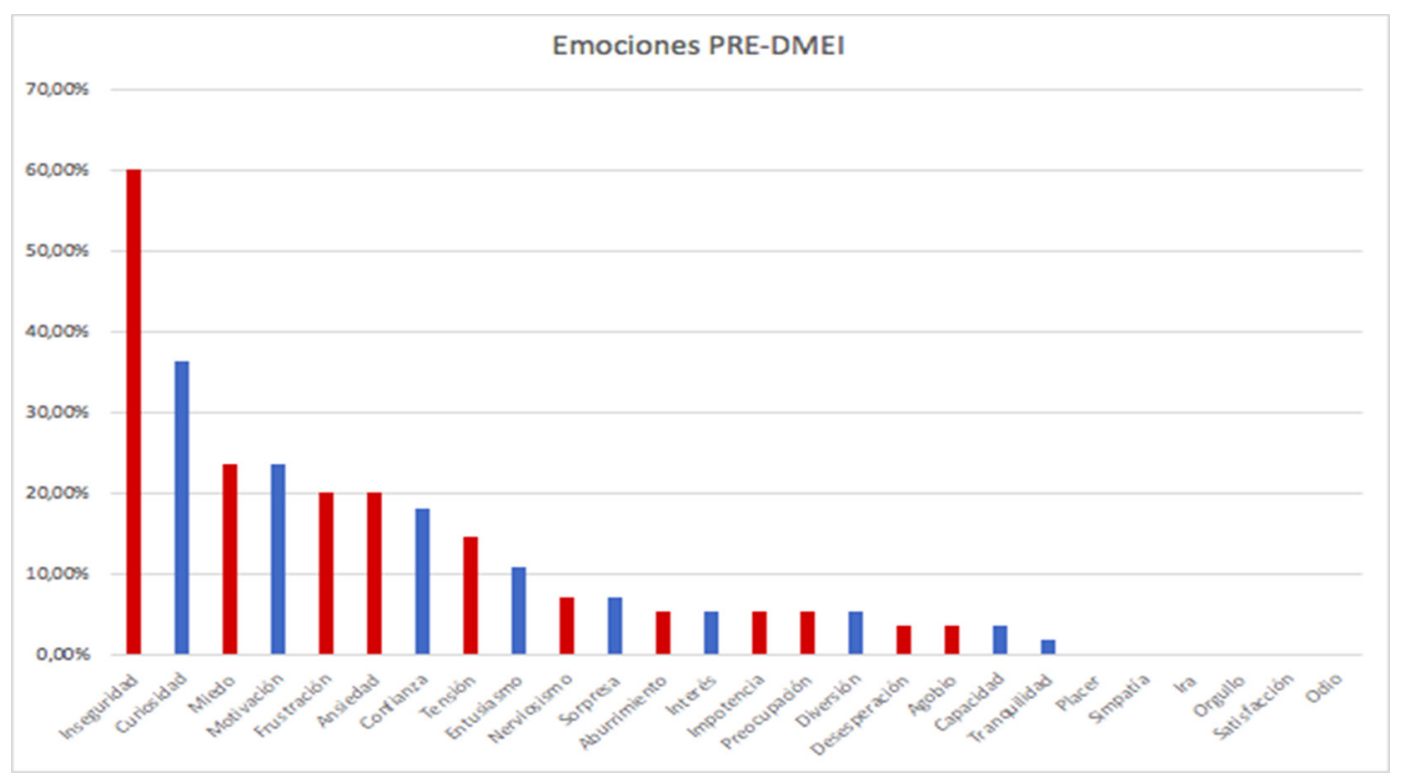

Figura. 1. Porcentajes de emociones positivas y negativas mencionadas antes de DMEI

Ante las preguntas relacionadas con las inquietudes y expectativas que tienen los alumnos sobre la asignatura, muchas de sus respuestas hacen referencia a la esperanza de cambiar las malas experiencias vividas en la etapa de educación obligatoria, pero además, insisten en la inseguridad de poder lograr los objetivos académicos de la asignatura. Tal como se recupera en el siguiente ejemplo, una alumna dice:

"Como hace muchos años que no estoy en contacto con la rama científica, me da un poco de respeto, dado que la experiencia previa no fue muy satisfactoria. Relacionado con esto, me preocupa que haya contenidos que se den por sabidos y me sienta perdida durante las clases." [13_3A]

Una alumna, por ejemplo, menciona varias emociones negativas en un solo comentario, con lo que se puede observar la evidente sensación de frustración que experimenta en 
relación con la dificultad, según ella, de trabajar contenidos científicos. Además, se hacen evidentes sus "buenas" intenciones relacionadas con los resultados (suponemos que relacionados con aprobar) mas no con el aprendizaje o cambio de actitud frente a las ciencias:

\begin{abstract}
"No hago esta asignatura desde segundo de la ESO, además, siempre he sufrido mucho para poder aprobarla (he tenido que recibir clases particulares) y aun así, no siempre he conseguido aprobarla. Estoy muy nerviosa pero haré todo lo que pueda para obtener buenos resultados" [7_5D]
\end{abstract}

Aunque en menor porcentaje, pero con una importante relevancia, encontramos también alumnos que expresan sus emociones desde una perspectiva optimista hacia la asignatura y que no por ello dejan ver cierto desencanto en relación con los "tópicos" de la física y la química:

Me gustaría aprender cosas nuevas y no tópicas sobre la física y la química. Quiero sorprenderme! [18_3A]

Encontrar este tipo de respuestas permite presentar una visión más optimista y mostrar al resto de la clase la posibilidad de encontrar espacios para promover el aprendizaje a partir de la curiosidad, la sorpresa, etc.

\title{
Etapa final: después de cursar DMEI
}

Las respuestas recogidas en el cuestionario final (Fig. 2) nos muestran un cambio considerable en relación con la etapa inicial. El cambio más destacable se refiere a la inseguridad, que solo es mencionada en un $7 \%$ de las respuestas, y a la curiosidad, que aumenta de un $36 \%$ a un $58 \%$.

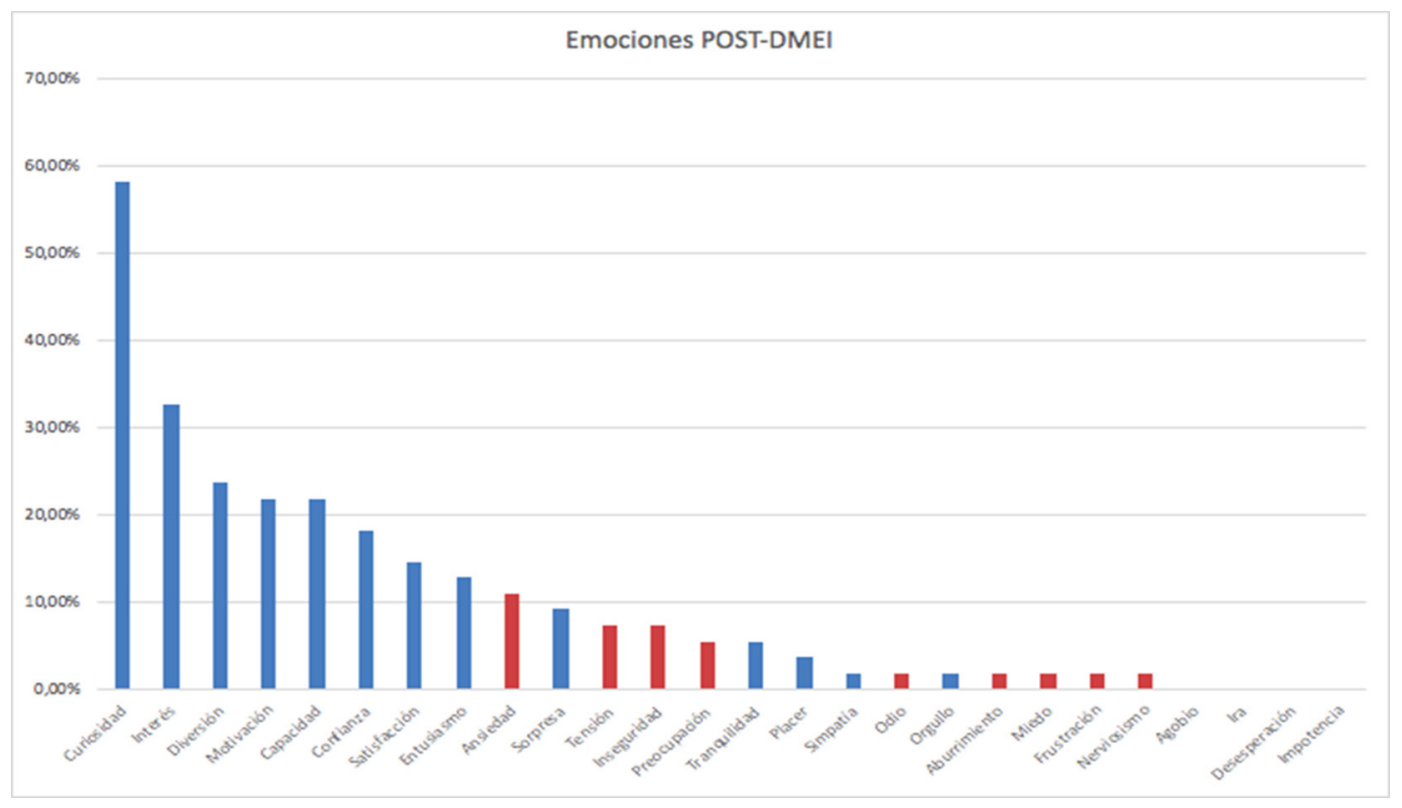

Figura. 2. Porcentajes de emociones positivas y negativas mencionadas después de DMEI

También destacamos la diversidad de emociones positivas que mencionan los alumnos después de haber cursado la asignatura y el incremento de su frecuencia en el discurso, como es el caso del interés, que en la etapa final llega a un 32,7\%. Otra emoción que evidencia el cambio de actitud es la diversión con un $23,6 \%$ después de haber cursado la asignatura y que antes no superaba el $10 \%$. 
Las emociones negativas disminuyen significativamente, siendo casi un $60 \%$ en la positiva con mayor porcentaje y solo un $10 \%$ en la negativa más mencionada.

Valoramos también positivamente el cambio en la sensación de capacidad, que pasa de un valor inferior al $5 \%$ a un valor superior al $30 \%$; y que es de especial relevancia si se compara con el porcentaje de la inseguridad, de casi un $60 \%$ en el formulario inicial e inferior al $10 \%$ en el final. Uno de los principales objetivos de la asignatura es capacitar a FM y disminuir su inseguridad, y en este sentido, el análisis nos permite evidenciar resultados satisfactorios, aunque siempre mejorables.

\section{Justificación del cambio de actitud}

A partir de las cuatro categorías identificadas como agentes motivadores del cambio emocional hemos identificado los siguientes resultados:

\section{A) La metodología de aula como principal agente motivador del cambio}

Un $85,5 \%$ de los alumnos reconoce haber cambiado las emociones después de cursar la asignatura. Entre las causas que argumentan encontramos que un $63 \%$ de los alumnos mencionan la metodología utilizada en DMEl como la principal razón para este cambio.

"La manera de hacer en las clases y la dinámica de la asignatura, tanto parte práctica como teórica me han hecho ver el mundo de la física desde otro punto de vista." [13-3A]

La mayoría de los FM que mencionan la palabra "teoría" la relacionan con sentimientos negativos, sobre todo antes de cursar la asignatura. En la justificación del cambio muchos mencionan que la metodología es el aspecto que les ha hecho modificar sus emociones respecto a las ciencias. Hacen referencia a que la metodología utilizada ha favorecido la significatividad de los contenidos trabajados, y ha cambiado su visión de una ciencia sólo teórica y alejada de la realidad a una ciencia más cercana y útil.

"...hace que todo aquello que no habías querido conocer comience a tener sentido" [2_5D]

Otro aspecto por destacar es como consiguen apropiarse de la idea de que teoría y práctica deben ser abordadas de manera conjunta:

"La unión entre teoría y práctica, y como nos han explicado conceptos que vemos difíciles de manera sencilla." [21_5D]

\section{B) El trabajo experimental y manipulativo como agente facilitador del cambio emocional}

El trabajo experimental también ha favorecido el cambio ya que un $48 \%$ de los alumnos hacen referencia a ello. Este tipo de actividades ponen de manifiesto la importancia de la implicación del alumno en el proceso de construcción del conocimiento.

"Esperaba que fuera una asignatura más teórica, y he visto que a través de la práctica he aprendido muchas cosas que no me imaginaba, y el hecho de compartir las ideas previas entre todos me ha servido para aprender de los demás y luego comprobar si los conocimientos previos que teníamos eran correctos y si no modificar nuestro conocimiento." [31_5D]

Además, hacen referencia a cómo la experimentación les ayuda a modificar sus ideas previas y reconstruir sus conocimientos a partir de observar y de aprender a plantearse preguntas:

"Poder identificar los conocimientos previos y llevar a cabo un procedimiento para deconstruirlos y construirlos desde la ciencia. Poder explicar conceptos mediante la experimentación, observación y hacerse preguntas." [26_3A] 


\section{C) Los vínculos docente-alumno como promotor del cambio emocional}

Entre otros argumentos que mencionan los FM (un 32\%), encontramos la actitud del docente como facilitador del cambio.

"A las facilidades que nos ha dado la maestra para entender el temario y comprender todos aquellos conocimientos que no creía posibles entender y aprender." [19_5A]

Dentro de los vínculos establecidos entre el docente y los FM recuperamos también la idea de cómo favorece el cambio el poder crear espacios de reflexión en el aula, en donde el alumno concibe como una oportunidad de aprendizaje el tiempo de reflexión sobre lo que se está trabajando en el aula.

"Creo que el cambio ha sido gracias a la metodología de clase, también por el profesor y porque se nos ha dado la oportunidad de pensar, observar y descubrir lo que nos estaban enseñando". [43_D2]

Con estos ejemplos se puede observar la importancia que comporta el tener empatía con los alumnos y disponer de diferentes recursos para promover la construcción de conocimiento científico, que es a lo que los alumnos se refieren cuando utilizan las palabras aprender y entender.

\section{D) La despenalización del error como herramienta facilitadora del cambio emocional}

Algunos alumnos (un 20\%) mencionan como relevante el hecho de que los docentes no penalicen el error, utilizándolo como punto de partida para modificar y construir sus conocimientos, así como también la posibilidad de aumentar su seguridad personal.

"La forma en que se ha impartido la clase y el hecho de no penalizar el error, dejando un espacio donde cada uno puede aportar su opinión sin miedo. Eso ha favorecido mejorar mi seguridad" [23_1A]"

También el hecho de poder construir conocimiento a partir de modificar sus ideas previas erróneas.

"He reestructurado aprendizajes erróneos y he observado diferentes métodos para plasmar de una forma más clara lo aprendido" [23_1A]

Con estos ejemplos observamos que el error sirve como punto de partida para generar espacios que promueven la construcción de conocimiento y que los mismos FM son conscientes de ello.

\section{Conclusiones}

A partir de los objetivos que nos habíamos planteado podemos concluir que los FM identifican y explicitan principalmente emociones negativas con relación a la física y la química al inicio del curso, modificando favorablemente dichas emociones al terminar la asignatura.

Una de las conclusiones más relevantes de este trabajo es la importancia de explicitar las emociones de los FM como punto de partida para poder reflexionar sobre ellas durante todo el proceso de enseñanza aprendizaje, con el objetivo de favorecer una modificación en la actitud hacia las ciencias, como así también una mejora en la autorregulación, el rendimiento académico y la motivación asociada a las emociones. Como docentes considera- 
mos que para que los cambios perduren y se consoliden, es imprescindible que se hagan explícitos y se valoren conjuntamente, y de aquí la importancia de dedicar momentos a presentar y explicitar los cambios emocionales entre el inicio y el final de curso.

Otro de nuestros objetivos era identificar y explicitar las causas que promueven el cambio emocional en los FM. En cuanto a esto, hemos podido identificar algunos aspectos de tipo metodológico y otros de tipo afectivo/social. Esta identificación, que ha permitido generar las categorías al menos en nuestro contexto, favorecerá su contrastación, redefinición y/o matización en futuros estudios, ya que en las clases es posible focalizar directamente estos aspectos.

Entre los aspectos metodológicos podemos destacar la valoración que hacen de llevar a la práctica clases participativas y dinámicas en dónde el rol del profesor es evidenciar la diferencia entre las ideas iniciales del alumnado y el conocimiento científico escolar, cuyo objetivo final es promover espacios de debate para conseguir una evolución de las representaciones idiosincráticas de los alumnos hacia los modelos científicos escolares que pueden ser significativos en las edades de la educación primaria. En estos aspectos metodológicos están relacionadas, por un lado, las actividades dialógicas (el planteo de preguntas investigables, los espacios de reflexión y la confrontación de ideas entre pares) y, por otro lado, la relevancia de la experimentación.

La experimentación ha permitido que los FM consigan conectar la teoría con la práctica, poniendo en evidencia ese conflicto entre lo que creen que sucede y las explicaciones que le damos al fenómeno en términos científicos. El trabajo de contenidos a partir de la experimentación también favorece de manera positiva la autoconfianza de los alumnos y por tanto el poder invertir en ellos la sensación de no tener capacidades para hacer ciencias o de ver la ciencia como algo lejano y difícil de realizar. El proponer en el aula experimentos, la mayoría de los cuales permiten dar sentido a situaciones cotidianas, desde una perspectiva científica, ha favorecido el cambio de la idea de la ciencia desvinculada de la vida diaria, permitiendo de esta manera que la mayoría de los FM pierdan el miedo y la inseguridad de abordar contenidos científicos. Es decir, se observa un cambio de una percepción de la física y la química "teóricas", desvinculadas de sus vidas, a una visión en la que los conocimientos adquieren sentido.

En relación con los aspectos más afectivos/sociales que favorecen este cambio en la percepción de la ciencia, hemos podido identificar que valoran el vínculo entre el profesorado y los FM.

Podemos concluir que el vínculo establecido en clase es un factor tan relevante como los demás aspectos comentados. Los FM explicitan, al comenzar las clases, recuerdos muy negativos y poco motivadores de sus clases de ciencias, siendo esto un desafío para el profesorado, ya que la investigación cada vez va consolidando más la idea de que es necesario establecer un buen clima de trabajo en el aula para favorecer el aprendizaje.

De la experiencia podemos decir que el vínculo que favorece este cambio de percepción relacionado con las ciencias se basa fundamentalmente en: atender a la diversidad de la clase, promover entre los alumnos espacios de reflexión conjunta, de tolerancia y respeto entre compañeros que evidencian diferentes niveles dominio de la asignatura y mostrar empatía con los FM y promoverla entre ellos.

También hemos podido observar que los espacios de clase en donde el profesorado hace explicitas las emociones que surgen en determinados momentos (como evidenciar la angustia de no entender los circuitos eléctricos, la alegría de conseguir encender una lamparita, o mejor aún, de entender cómo es que esto sucede, así como de hacer nue- 
vas predicciones) permiten que sientan que están haciendo ciencia de manera cercana y asequible. Esto provoca que asimilen sus emociones hacia la disciplina como algo normal y propio del proceso de enseñanza-aprendizaje.

Otro aspecto que ha favorecido el cambio ha sido el uso del error como promotor de la construcción del conocimiento. El miedo a equivocarse es uno de los principales obstáculos y en el ámbito científico existen varios mitos relacionados con la necesidad de ser brillantes para entender ciencias. Esta visión promueve la baja autoconfianza de los estudiantes en las asignaturas de ciencias. Este miedo se transforma cuando los FM son conscientes de la importancia de utilizar el error como punto de partida, no para avergonzar a los alumnos, sino como una herramienta para generar un conflicto que nos ayude a construir conocimiento.

A pesar de los resultados alentadores que hemos obtenido en cuanto al cambio de las emociones y su relación con el enfoque didáctico y la metodología de clase, encontramos que algunos FM muestran todavía una visión negativa de la teoría, relacionándola casi exclusivamente con las fórmulas y las definiciones, sin acabar de comprender que los esfuerzos que ellos mismos han hecho, y han valorado positivamente, de construir modelos de ciencia escolar a partir de la interacción con los fenómenos y con sus pares, es elaboración teórica. Es necesario que ahondemos esfuerzos en ayudar a los FM a ser conscientes de sus propias elaboraciones teóricas y de la satisfacción que pueden encontrar en este proceso, de tal manera que no vean la "teoría" solo como un aspecto que se puede relacionar con la práctica en las clases, sino que los dos aspectos, teoría y práctica, son indisolubles en la actividad científica escolar. Por otro lado, algunos alumnos no logran relacionar lo que aprenden en clase con problemas de la vida cotidiana que puedan abordarse a partir de la experimentación. Para favorecer esta conexión contexto-teoría-práctica, nos proponemos incorporar en la práctica científica escolar, actividades experimentales contextualizadas en las cuales los alumnos diseñen experimentos, en los que se aproveche el potencial didáctico de herramientas digitales como sensores conectados a través de tabletas o smartphones.

Investigación financiada por el Ministerio de Economía y Competitividad (con referencia EDU2015-66643-C2-1-P) y realizada en el marco del grupo consolidado ACELEC reconocido por la AGAUR (con referencia 2017SGR1399).

\section{Bibliografía}

Appleton, K. (1995). Student teachers' confidence to teach science: is more science knowledge necessary to improve self-confidence? International Journal of Science Education, 17(3), 357-369. DOI: https://doi.org/10.1080/0950069950170307

Borrachero Cortés, A. (2015). Las emociones en la enseñanza y el aprendizaje de las ciencias en Educación Secundaria. Tesis doctoral. Universidad de Extremadura. Recuperado de: http://dehesa.unex.es/handle/10662/3066

Cañal, P. (2000). El conocimiento profesional sobre las ciencias y la alfabetización científica en primaria. Alambique, 24, 46-56.

Dávila Acedo, M. A., Cañada Cañada, F., Sánchez Martín, J., y Mellado Jiménez, V. (2016). Las emociones en el aprendizaje de física y química en educación secundaria. Causas relacionadas con el estudiante. Educación química, 27(3), 217-225. DOI: https://doi.org/10.1016/j.eq.2016.04.001 
Gess-Newsome, J. (2002). Secondary Teachers' Knowledge and Beliefs about Subject Matter and their Impact on Instruction. En J. Gess-Newsome y N. G. Lederman (Eds.), Examining Pedagogical Content Knowledge (Vol. 6). Dordrecht: Kluwer Academic Publishers.

Harlen, W., y Holroyd, C. (1997). Primary teachers' understanding of concepts of science: impact on confidence and teaching. International Journal of Science Education, 19(1), 93-105. DOI: https://doi.org/10.1080/0950069970190107

Hollon, R., Roth, K., y Anderson, C. (1991). Science teachers' conceptions of teaching and learning. En Brhopy, J. (Ed.), Advances in research on teaching (Vol. 2, pp. 145-186). Greenwich (CT): JAI Press.

Hugo, D. V. H., Sanmartí, N., y Adúriz-Bravo, A. (2013). Estilos de trabajo emocional del futuro profesorado de ciencias durante el practicum. Enseñanza de las ciencias, 31(1), 151-167. DOI: https://doi.org/10.5565/rev/ec/v31n1.606

Mega, C., Ronconi, L., y De Beni, R. (2014). What makes a good student? How emotions, self-regulated learning, and motivation contribute to academic achievement. Journal of Educational Psychology, 106(1), 121-131.

Mellado, V. (2003). Cambio didáctico del profesorado de ciencias experimentales y filosofía de la ciencia. Enseñanza de las Ciencias, 21(3), 343-358.

Mellado, V. M., Borrachero, A. B., Brígido, M., Melo, L. V., Dávila, M. A., Cañada, F., y Al, E. (2014). Las emociones en la enseñanza de las ciencias. Enseñanza de las ciencias, 32(3), 11-36. DOI: https://doi.org/10.5565/rev/ensciencias.1478

Murphy, C., y Beggs, J. (2003). Children's perceptions of school science. School Science Review Number 308 - The Association for Science Education, 84(308), 109-116.

Osborne, J., Driver, R., y Simon, S. (1998). Attitudes to Science: Issues and Concerns. School Science Review, 79(288), 27-33.

Parkinson, J., Hendley, D., Tanner, H., y Stables, A. (1998). Pupils' Attitudes to Science in Key Stage 3 of the National Curriculum: a study of pupils in South Wales. Research in Science y Technological Education, 16(2), 165-176. DOI: https://doi. org/10.1080/0263514980160206

Pérez Serrano, G. (1994). Investigación cualitativa. Retos e interrogantes I Métodos. Madrid: La Muralla.

Pipitone, C., LLadó, À. G., Mas, J. G., Mingo, A. C. de, Carvajal, I. M., Agudelo, C., y Hernández, E. M. (2016a). Actividades dialógicas de ciencias en la formación inicial del profesorado de educación primaria. Dialogical activities of science in the initial teacher training of Primary Education. Campo Abierto. Revista de Educación, 35(1), 93-108.

Ramsden, J. M. (1998). Mission impossible?: Can anything be done about attitudes to science? International Journal of Science Education, 20(2), 125-137. DOI: https://doi. org/10.1080/0950069980200201

Shapiro, S. (2010). Revisiting the teachers' lounge: Reflections on emotional experience and teacher identity. Teaching and Teacher Education, 26(3), 616-621.

Talanquer, V. (2004). Formación docente: ¿Qué conocimiento distingue a los buenos maestros de química? Educación química, 15(1), 52-58.

Tesch, R. (1990). Qualitative Research: Analysis Types and Software Tools. New York: Falmer Press. 
Vázquez Alonso, Á., y Manassero-Mas, M. A. (2008). El declive de las actitudes hacia la ciencia de los estudiantes: un indicador inquietante para la educación científica. Revista Eureka sobre Enseñanza y Divulgación de las Ciencias - 2008, 5(3) pp. 274292.

Zembylas, M. (2001). Constructing genealogies of teachers' emotions in science teaching - Zembylas - 2001 - Wiley Online Library. Journal of Research in Science Teaching, (39), 79-103. DOI: https://doi.org/10.1002/tea.10010

Zembylas, M. (2003). Emotions and Teacher Identity: A poststructural perspective. Teachers and Teaching, 9(3), 213-238. DOI: https://doi.org/10.1080/13540600309 378 